\title{
Does thyroid surgery for Graves' disease improve health-related quality of life?
}

\author{
Gregorio Scerrino • Giuditta Morfino • Nunzia Cinzia Paladino • \\ Valentina Di Paola $\cdot$ Emanuele Amodio - Gaspare Gulotta • \\ Sebastiano Bonventre
}

Received: 3 May 2012/ Accepted: 24 September 2012

(C) Springer Japan 2012

\begin{abstract}
Purpose Graves' disease can induce alterations of the psychosocial well-being that negatively influence the overall well-being of patients. Among the current treatments, surgery has limited indications, and its impact on the health-related quality of life has not been well clarified. The aim of this study was to assess the impact of surgery on the quality of life.

Methods Fifty-seven patients who underwent total thyroidectomy for Graves' disease in our surgical unit between April 2002 and December 2009 were administered a questionnaire concerning four issues: organic alterations and clinical manifestations, neurovegetative system disturbances, impairment of daily activities, psychosocial problems. Patients were retrospectively questioned after thyroidectomy about the presence of these symptoms in both the pre and postoperative periods.

Results There was a significant improvement after surgery in all four areas. Organic manifestations and psychosocial problems had higher average improvements, as did some aspects of the neurovegetative system and difficulties in undertaking daily activities. There were no reports of a worsening of symptoms.

Conclusions Surgery resolved the hyperthyroidism in $100 \%$ of cases, and was associated with a quality of life
\end{abstract}

G. Scerrino $(\varangle) \cdot$ G. Morfino - N. C. Paladino · V. Di Paola •

G. Gulotta $\cdot$ S. Bonventre

Unit of General and Emergency Surgery, Department of General, Emergency and Transplant Surgery (GENURTO), University of Palermo, Via Alcide De Gasperi 53, 90146 Palermo, Italy e-mail: gregorio.scerrino@tiscali.it

E. Amodio

Department of Sciences for Health Promotion

"G. D'Alessandro", University of Palermo, Palermo, Italy improvement of about $70 \%$ in the patients. Surgery can therefore provide an immediate and effective resolution of Graves' disease, with benefits in health-related quality of life.

Keywords Graves' disease · Quality of life · Questionnaire · Total thyroidectomy

\section{Introduction}

Graves' disease (GD) is an autoimmune thyroid disorder characterized by the presence of immunoglobulins in the blood that stimulate the thyroid and bind to thyrotropin receptors, leading to hyperfunction of the gland and consequent excessive secretion of thyroid hormones. GD is the most common cause of hyperthyroidism. The incidence of GD is estimated at 5 per 1,000 per year, with a femaleto-male ratio of 7:1 [1]. Patients with untreated GD suffer from a wide range of symptoms, and a substantial portion of them experience limitations in their usual activities, perceive their general health as impaired, and experience social and emotional deterioration [2]. Thus, the impact of GD on the overall well-being of patients cannot be neglected, and some studies have shown that patients with the disease can have a diminished health-related quality of life (HRQOL) [2, 3]. As a result, the importance of taking the HRQOL into consideration when evaluating thyroid patients has become increasingly recognized as one of the main goals of the various disease treatments [4].

To date, pharmacological therapy (methimazole, propylthiouracil, carbimazole) is the principal strategy used in many European countries (77 \%) and in Japan (88 \%) [5], while radioactive iodine therapy is usually recommended in North America (70 \%) [6, 7]. Surgical therapy 
undoubtedly leads to an immediate (100\% of cases [6]) and permanent (92\% of cases [8]) resolution of hyperthyroidism, and reduces the possible progression of ophthalmopathy, resulting in a rapid clinical improvement. It also minimizes the risk of malignancy in patients with concomitant GD and thyroid nodules [9].

Despite these benefits, patients with GD are rarely indicated for surgery, and the impact of surgery on the HRQOL has not yet been well documented [10]. In fact, the HRQOL has been studied principally in the setting of orbitopathy $[11,12]$, where it seems that a worsening HRQOL is related to the grade of ophthalmopathy [12]. However, no studies have so far focused on the impact of surgery on the subsequent HRQOL in GD patients.

The aim of our study was therefore to assess the effectiveness of surgical therapy on one of the most important aspects of the disease, namely, the quality of life of individual patients.

\section{Materials and methods}

This study was retrospectively carried out on 57 of 62 patients who had undergone total thyroidectomy for Graves' disease (diagnosis confirmed by histology) at the Operative Surgical Unit of P. Giaccone University Hospital, in Palermo, Italy from April 2002 to December 2009.

In order to avoid an important bias, we recruited patients with no or mild orbitopathy, excluding those who presented proptosis, and those with preoperative hyperparathyroidism. In addition, we excluded five patients: three because of an incidental coexistent papillary carcinoma on final pathology, and two who were untraceable during active recruitment. The diagnosis of GD was made by assessment of the clinical features, an antibody analysis, and ultrasound scanning. Our indications for surgery were similar to the generally accepted indications: large goiters with compressive symptoms (19/57 in our group); intolerance or failure of medical therapy (23/57); recurrent hyperthyroidism (28/57); co-existence of nodules with ultrasound features and/or biopsy suspicious for malignancy (3/60, all excluded from the study because of confirmed malignancies); history of irradiation to the head and neck (1/57); patients preferring rapid resolution of their discomfort (5/57).

The patients had been operated on by the same surgical team (two of three expert surgeons, G.G., G.S. and N.C.P., treated each patient) and were followed up periodically at our Endocrine Surgery Unit. The postoperative hospital stay after the operation was 1 day in 50 cases, and 2 days in seven cases, for an average of 1.1 days. From April to June 2008 and from February to June 2010, a standardized questionnaire was administered retrospectively to each patient by a single individual (a person on the surgical team already known to the patient). The questionnaire was administered between 6 months and 6 years after surgery, with a median of 3 years (range 5-49 months). After obtaining informed consent from each patient, and official approval from the Internal Review Board of our hospital, each interview with the questionnaire was conducted during a mean time of $15 \mathrm{~min}$. The study protocol conformed to the ethical guidelines of the 1975 Declaration of Helsinki and was in accordance with Italian regulations. Ethics committee approval was not required for this study.

To compare the results obtained in terms of quality of life and to provide a control group, we administered the same questionnaire from February to June 2010 to a group of patients recruited from the Endocrine Surgery Unit of our hospital who had received medical therapy with methimazole, each of them for a different time period, with a mean of 30 months (range 12-48 months). There were 25 patients in the control group, none of whom had undergone surgery: four males $(16 \%)$ and 21 females $(84 \%)$ with mean age of 46 years. The mean thyroid volume was $36.9 \mathrm{ml}$, and the patients had been affected with GD for a period ranging from 2 to 7 years. This control group was necessarily smaller because the patients were matched for the same features as the surgery group (age, gender, occupation and thyroid volume).

\section{Questionnaire}

Based on the most frequently observed symptoms and questionnaires found in the international literature (Medical Outcomes Study-24 questionnaire [13]; Short Form-36 questionnaire [14]; EuroQol VAS Questionnaire [15]; National Eye Institute Visual Function Questionnaire [16]; Profile of Mood States [17]; Graves' ophthalmopathy Quality of Life Questionnaire-GOQOL [18]), we developed a questionnaire to measure the HRQOL in patients who had undergone total thyroidectomy for GD. Many of the questions included in our questionnaire were derived from these previously cited studies and were thus considered to be validated. The questionnaire was pilot tested on 10 patients to evaluate its repeatability (test/retest reliability), readability, and comprehension of each item. The final version of the questionnaire included 36 items organized into 5 different sections:

1. Private data and social-demographic information $(n=4)$ :

- age

- gender

- marital status

- occupation 
2. Organic and clinical manifestations $(n=6)$ :

- deterioration of visual faculties (conjunctivitis, diplopia, lack of ocular convergence, decreased visual acuity, blurred vision, chemosis, irritation, tearing, spontaneous retro-bulbar pain and pain with ocular movement, photophobia, ocular foreign body sensation)

- objective changes in the face and body (proptosis, lagophthalmos, eyelid retraction or extension, ocular erythema, swelling of the lachrymal caruncle, edema of the face, hands, feet and periorbital region, weight changes, fragility of the skin, nails and hair, change in voice, enlarged tongue, gynecomastia)

- cardiovascular problems (blood pressure and heart rate alteration, palpitations, fibrillation)

- gastrointestinal problems (difficulty swallowing, sensation of postprandial fullness, diarrhea or constipation, vomiting, nausea)

- respiratory disorders (dyspnea, chest pain, pharyngitis, bronchitis)

- skeletal and muscular alterations (hand tremors, fatigue, pain, adynamia, asthenia).

All the items were measured on a three-point scale, categorized as 0 ("none"), 1 ("some"), and 2 ("many"). For each patient, a sub-score was created by averaging the scores for the six items.

3. Aspects of the neurovegetative system and other general alterations related or not to thyroid disorders $(n=6)$ :

- disorders of the peripheral nervous system (heat or cold intolerance, dizziness, altered sense of hearing, smell, taste)

- alteration of physiological sweating

- increased or decreased appetite

- menstrual disturbances

- sexual problems (impotence and/or decreased libido)

- headache

All the items were measured on a three-point scale, categorized as 0 ("none"), 1 ("some"), and 2 ("many"). For each patient, a sub-score was created by averaging the scores of the six items.

4. Daily activities $(n=7)$ :

- driving a car

- reading a newspaper

- working

- performing household chores (cooking, moving indoors or outdoors)

- watching TV
- engaging in a hobby

- walking or cycling or using public transport. All the items were measured on a three-point scale, categorized as 0 ("no limitations"), 1 ("moderate limitations"), and 2 ("severe limitations"). For each patient, a sub-score was created by calculating the average of the scores for the seven items.

5. Psychological and social problems $(n=12)$ :

- emotional lability

- sleep disorders (insomnia)

- hyperactivity, anxiety or nervousness

- depression

- loss of self confidence

- stress

- decreased attention and/or memory span

- decreased capacity for planning and/or solving complex problems

- hallucinations

- social isolation (difficulty in making friends, feeling continuously observed, appearing less frequently in photographs)

- using camouflage to change self appearance

- observing any change in attitude on the part of others towards the patient (unpleasant answers, a lack of availability).

All the items were measured on a three-point scale, categorized as 0 ("no limitations"), 1 ("moderate limitations") and 2 ("severe limitations"). For each patient, a sub-score was created by averaging the scores of the 12 items.

6. Scar quality $(n=1)$ as perceived by the patients and assessed as "excellent," "good," or "poor."

Items in sections $2-5$ of the questionnaire investigated the HRQOL, both as retrospectively recalled by patients about their status before surgery and as the HRQOL at the time of the interview. Lower HRQOL scores indicated better health.

The thyroid volume was obtained from the patients' medical records, then by ultrasound, and subsequently by the final pathological examination. We evaluated the conditions of hormone compensation (dose of thyroid hormones, parathyroid hormone (PTH), and values of TSH receptor antibodies, or TRAb) (Table 4). All patients were confirmed to be in a condition of hyperthyroidism when the surgical option was proposed, and underwent total thyroidectomy in a state of clinical-biological euthyroidism after 1-2 months of medical therapy (methimazole). In addition, since the patients with preoperative hyperparathyroidism were excluded and there was no alteration in either the preoperative (hyperparathyroidism) or postoperative (iatrogenic hypoparathyroidism) levels, it was not necessary to investigate vitamin $\mathrm{D}$ values. 
We usually follow our patients for up to 12-18 months after surgery through clinical, laboratory (dose of serum calcium levels, thyroid hormone levels and TRAb) and instrumental (ultrasound scanning) evaluations. We performed examinations with a fiberoptic laryngoscope in cases with vocal cord alterations. The median interval between symptom onset and the surgery for these patients was 12 months (range 4-48 months).

\section{Statistical analysis}

The questionnaire responses were entered into an electronic worksheet (Microsoft Excel). Absolute and relative frequencies were calculated for qualitative variables, while quantitative variables were summarized as the mean \pm standard deviation or medians (range). The means and $95 \%$ confidence intervals (95\% CIs) were also calculated.

Quantitative variables were compared with the paired or unpaired Student's $t$ test, ANOVA or the Mann-Whitney $U$ test, as appropriate. Statistical significance for all analyses was established as a two-tailed $p$ value of 0.05 . All the data were analyzed using the $\mathrm{R}$ statistical software package [19].

\section{Results}

The characteristics of the 57 patients, comprising nine males $(16 \%)$ and 48 females $(84 \%)$, with a mean age of $47.7 \pm 13.7$ years, are shown in Table 1 . Of the study participants, $37 \%$ were employed, in a variety of occupational sectors. The mean thyroid volume was $34.7 \pm 15 \mathrm{ml}$.

As shown in Fig. 1, before surgery, organic manifestations were the most significant problems, with an average score of 1.1. The psychosocial and neurovegetative

Table 1 Patient characteristics

\begin{tabular}{lr}
\hline & $n=57$ \\
\hline Age in years, mean (SD) & $47.7(13.7)$ \\
Gender, $n(\%)$ & $9(15.8)$ \\
Male & $48(84.2)$ \\
Female & \\
Occupation, $n(\%)$ & $10(17.6)$ \\
Unemployed/student & $14(24.5)$ \\
Housewife & $21(36.8)$ \\
Employed, different sectors & $12(21.1)$ \\
Retired & $34.7(15)$ \\
Thyroid volume in ml, mean (SD)
\end{tabular}

$n$ total number of patients problems were perceived to be moderately limiting (average score per item $=0.63$ ), whereas difficulties in daily activities were considered to be not very limiting (average score per item $=0.22$ ). Among the different symptoms/ problems, those associated with severe limitations were cardiovascular alterations (in 37 patients, $65 \%$ ), skeletal and muscular alterations (in 35 patients, 61\%), physiological sweating (in 23 patients, $40 \%$ ), and objective changes in the face and body (in 22 patients, $39 \%$ ).

All the investigated symptoms/signs had a significant improvement 3-6 months after surgery. The organic/clinical manifestations and psychosocial problems had higher average improvements than the other issues examined (from 1.1 to 0.28 , and from 0.63 to 0.19 , respectively; $p<0.001$ in both cases). Neurovegetative system problems and difficulties in daily activities had lower, but still significant, improvements (from 0.63 to 0.20 , and from 0.22 to 0.06 , respectively; $p<0.001$ in both cases). Based on the total score of organic alterations and clinical manifestations, neurovegetative system disturbances, limitations of daily activities and psychosocial problems, there was a shift from 0.63 before surgery to 0.18 after surgery $(p<0.001)$.

The ten main alterations/problems affecting the HRQOL before surgery are summarized in Table 2. Organic problems and clinical manifestations (cardiovascular disorders, skeletal and muscular alterations, objective changes in the face and body) were the three items with the highest scores. These ten items all had significant improvements following surgery, ranging from 61 to $86 \%$ improvement.

Considering 31 items, after excluding the 4 questions included in part 1 and the sole item in part 6 , the social isolation, limited working capacity and cardiovascular alterations were those registering the highest improvements after surgery (93, 90 and $86 \%$, respectively) (data not shown).

None of the variables considered in this study (age, gender, occupation and thyroid volume) were significantly related to a higher improvement in the health-related quality of life (Table 3). The individual assessment of the final wound was excellent for 41 patients $(72 \%)$, good for $16(28 \%)$ and insufficient to none $(0 \%)$.

Regarding hormonal compensation, no patient had a recurrence of persistent hyperthyroidism; two patients had subclinical hypothyroidism despite replacement therapy, while none of the patients had clinical hypothyroidism. Fifty-six of the 57 patients $(98 \%)$ had no long-term complications or permanent hypocalcemia 6 months after surgery. None of the patients took long-term calcium replacement therapy (after 6 months); in all patients there was a gradual decrease in serum TRAb levels and no significant differences between the pre and postoperative PTH values, which were normal (Table 4). However, it should 
Fig. 1 The health-related quality of life (organic/clinical, psychosocial, neurovegetative, daily activities and total scores) in patients before and 3-6 months after surgery (lower scores indicate better health)

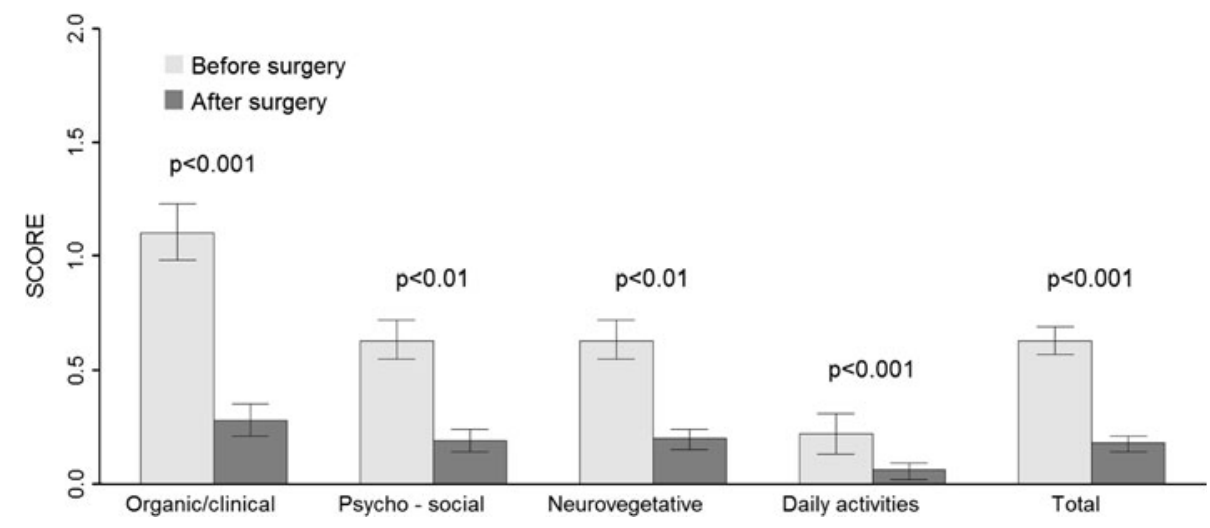

Table 2 The top ten alterations/problems affecting the healthrelated quality of life in patients with graves' disease before surgery, and 3-6 months after surgery

\begin{tabular}{llll}
\hline Alteration/problem & Category & $\begin{array}{l}\text { Score before } \\
\text { surgery }\end{array}$ & $\begin{array}{l}\text { Score after } \\
\text { surgery }\end{array}$ \\
\hline $\begin{array}{l}\text { Cardiovascular disorders } \\
\begin{array}{l}\text { Skeletal and muscular } \\
\text { alterations }\end{array}\end{array}$ & CM & 1.61 & 0.23 \\
$\begin{array}{l}\text { Objective changes in the } \\
\text { face and body }\end{array}$ & CM & 1.46 & 0.37 \\
$\begin{array}{l}\text { Alteration of } \\
\text { physiological sweating }\end{array}$ & NS & 1.11 & 0.32 \\
$\begin{array}{l}\text { Emotional lability } \\
\begin{array}{l}\text { Hyperactivity, anxiety or } \\
\text { nervousness }\end{array}\end{array}$ & PSS & 1.11 & 0.33 \\
$\begin{array}{l}\text { Gastrointestinal disorders } \\
\begin{array}{l}\text { Sleep disorders } \\
\text { Depression }\end{array}\end{array}$ & CM & 1.07 & 0.33 \\
$\begin{array}{l}\text { Increased or decreased } \\
\text { appetite }\end{array}$ & PSS & 0.98 & 0.30 \\
\hline
\end{tabular}

$C M$ clinical manifestations, $N S$ aspects of neurovegetative system, $P S S$ psychological and social problems

be borne in mind that the general consensus in the literature is that normalization of the autoimmune abnormality comes much later, after at least 18 months [20, 21]. One patient $(2 \%)$ in our study had transient unilateral vocal cord palsy detected by the fiberoptic laryngoscope, but without evident impairment of the voice.

With regard to the control group, in addition to the wellknown side effects and recurrences following medical therapy [22, 23], the most frequent problems that persisted despite anti-thyroid drug treatment concerned clinical manifestations (gastrointestinal, cardiovascular and muscular alterations), and also alterations in physical appearance, neurovegetative and psychological systems (anxiety, insomnia, depression and stress) and social disorders. Some improvement was observed in body weight and hormonal values at the beginning of therapy; although worsened ocular pressures, exophthalmos and migraine were also
Table 3 Average improvement in the health-related quality of life in patients, stratified by gender, age, occupation and thyroid volume

\begin{tabular}{lll}
\hline & $\begin{array}{l}\text { Average improvement } \\
\text { in health-related quality } \\
\text { of life } \pm \text { SD }\end{array}$ & $p$ value \\
\hline Gender & & \\
$\quad$ Male & $11.2 \pm 4.3$ & $0.18^{*}$ \\
Female & $14.5 \pm 7.1$ & \\
Age (years) & & \\
$<35$ & $16.7 \pm 8.3$ & \\
$35-49$ & $12.7 \pm 5.7$ & \\
$50-64$ & $13.8 \pm 6.5$ & $0.96^{* *}$ \\
$>65$ & $13.6 \pm 8$ & \\
Occupation & & \\
Unemployed/student & $15.1 \pm 8.3$ & \\
Housewife & $13.9 \pm 6.1$ & \\
Employed, different sectors & $13.7 \pm 7$ & \\
Retired & $13.8 \pm 7$ & \\
Thyroid volume (ml) & & \\
$<30$ & $14.2 \pm 7.7$ & \\
$30-39$ & $13.6 \pm 7.4$ & \\
$>39$ & $14.3 \pm 4.7$ & \\
\hline
\end{tabular}

* Unpaired Student's $t$ test

** ANOVA

observed (final results summarized in Table 5). Finally, the patients who underwent surgery had lower HQOL scores than patients $(n=25)$ who were treated with anti-thyroid drugs $(p<0.001)$ (Table 5). Therefore, compared with the control group, the patients who underwent surgery had a better QOL than those that received prolonged medical therapy.

\section{Discussion}

Graves' disease is the most common cause of hyperthyroidism (60-80 \%) [5] and thyrotoxicosis. It affects 
Table 4 The median serum antibody values during the pre and postoperative periods (12 months after surgery)

\begin{tabular}{lccc}
\hline Antibodies & Before surgery & $\begin{array}{l}\text { After surgery } \\
(12 \text { months })\end{array}$ & $p$ value $^{*}$ \\
\hline $\begin{array}{c}\text { TRAb (IU/L) } \\
\text { Median (range) }\end{array}$ & $3.2(0.3-13.3)$ & $1.4(0.2-8.0)$ & $<0.001$ \\
$\begin{array}{c}\text { TPO Ab (IU/mL) } \\
\text { Median (range) }\end{array}$ & $43.8(6.7-140.0)$ & $40.0(6.0-215.0)$ & $<0.01$ \\
$\begin{array}{c}\text { TG Ab (IU/mL) } \\
\text { Median (range) }\end{array}$ & $83.0(11.0-139.0)$ & $78.0(11.0-30.0)$ & $<0.001$ \\
$\begin{array}{c}\text { PTH (pg/mL) } \\
\text { Mean (standard } \\
\text { deviation) }\end{array}$ & $45.8(31-65)$ & $47.6(35-70)$ & 0.17 \\
\hline
\end{tabular}

$\overline{T R A b \text { TSH-receptor antibodies, TPO } A b \text { thyroid peroxidase antibod- }}$ ies, $T G A b$ thyroglobulin antibodies, $P T H$ parathyroid hormone

* Paired Student's $t$ test

Table 5 Average improvement in the health-related quality of life in patients who underwent surgery and in patients treated with antithyroid drugs

\begin{tabular}{|c|c|c|c|}
\hline \multirow{2}{*}{$\begin{array}{l}\text { Alteration/ } \\
\text { problem }\end{array}$} & \multicolumn{2}{|c|}{ Average score \pm SD } & \multirow[t]{2}{*}{$p$ value* } \\
\hline & $\begin{array}{l}\text { Patients who } \\
\text { underwent } \\
\text { surgery }\end{array}$ & $\begin{array}{l}\text { Patients treated with } \\
\text { anti-thyroid drugs }\end{array}$ & \\
\hline Organic/clinical & $0.28 \pm 0.27$ & $0.92 \pm 0.50$ & $<0.001$ \\
\hline Psychosocial & $0.20 \pm 0.20$ & $0.63 \pm 0.35$ & $<0.0001$ \\
\hline Neurovegetative & $0.20 \pm 0.16$ & $0.58 \pm 0.35$ & $<0.001$ \\
\hline Daily activities & $0.06 \pm 0.13$ & $0.11 \pm 0.32$ & 0.26 \\
\hline Total & $0.19 \pm 0.14$ & $0.56 \pm 0.24$ & $<0.001$ \\
\hline
\end{tabular}

* Unpaired Student's $t$ test

females 10 times more frequently than males, and overall, generally affects those between the ages of 40 and 60 years, but can also manifest in females of childbearing age [24]. The reported epidemiological features of Graves' disease were well represented in our study sample, allowing us to hypothesize a reasonable generalizability of our results.

Graves' disease is clinically characterized by a symmetrical goiter, hyperthyroidism and an infiltrative ophthalmopathy often associated with a dermopathy. Ophthalmopathy is the most common extrathyroidal manifestation of Graves' disease (50\% of cases) [25], and is a greatly limiting condition in patients. GD is also often associated with changes in physical appearance, especially in the eyes and face, which become disfigured, and alterations of the mental state, such as depression, anxiety, mania, cognitive dysfunction [26], and is sometimes associated with psychiatric and psychosocial disorders [27], such as confusion, tension, anger, inertia, hostility, inability to work, uncertainty of the future, unhappiness, panic episodes and isolation. All these factors may also weigh heavily on the psychological well-being of these patients by interfering with their daily activities [27]. Our findings confirm that, before surgery, Graves' disease patients have a diminished HRQOL, which involves all the aspects investigated in our study, in particular, as a consequence of the organic and clinical manifestations of the disease. Following surgery, the HRQOL still remained impaired, although a general improvement was evident, with improvements in sub-scores ranging from $69 \%$ (for aspects related to the neurovegetative system) to $75 \%$ (relating to organic manifestations). Overall, surgery was associated with an increase of about $70 \%$ in the HRQOL.

Although many of our questions were culled from the literature, our questionnaire was largely original and designed to be applicable to, and valid for, our patient population. As a result, our findings may not be fully comparable with those of other studies. However, the lack of a questionnaire that assesses the psychological status, as well as the general, clinical and psychosocial aspects of GD in all its complexity, as well as the response to medical and/or surgical treatment, is acknowledged in the international literature [2, 18, 28].

Surprisingly, our results showed that none of the patient characteristics were significantly associated with a higher HRQOL impairment after surgery. This finding suggests that surgical treatment achieves similar HRQOL benefits regardless of the patient age, gender and thyroid volume, so that even patients of older age or with a high thyroid volume should be considered likely to benefit from such therapy.

Finally, this study has important limitations that need to be kept in mind when interpreting the results, such as the retrospective administration and evaluation of the questionnaire, the subjectivity of the estimation of visual ability and cosmetic results, the limited sample size; use of anamnestic findings for some aspects (possible recall bias), the treatments performed by a single surgical team at a single center, and the long-time interval between surgery and the interview for some patients. We are well aware that it may have been better to administer the questionnaire preoperatively and then again after a defined period of time following thyroidectomy, but a prospective study would require a high number of patients, which our unit, performing an average number of thyroidectomies annually, would not be able to recruit in a reasonable time interval.

We performed a longitudinal study with a retrospective approach to preliminarily assess the role of surgery in improving the patient QoL. This study methodology is certainly not new, although some have found that "a method for measuring change in QoL incorporating a retrospective baseline-measurement showed strongest associations with change in clinical indicators of health status, suggesting a more valid measurement of change in 
QoL than a conventional prospective method" [29]. This retrospective approach is associated with the theory of "response shift," which revolves on the notion that patients' perceptions of, and expectations for, QoL shift over time, and that a retrospective analysis takes this shift into account more fully than does a prospective approach. The other school of thought, the "implicit theory of change," hinges on the idea that patients assume an implicit change in their present status and then try to gather impressions of a previous status based on their present state, thus introducing an important bias. The "implicit change" theorists stress the idea that only actual states of health can be measured, and that this can only be done prospectively. The "response shift" theorists claim that a prospective approach compromises any modification of personal expectation and perception on the patient's part. It is important to underscore the fact that the theory of "response shift" was prompted largely by studies that found patients' positive perceptions of their HRQOL objectively at odds with their poor health status. Although there is much debate over the most appropriate study design for measuring HRQOL, there have been no compelling studies comparing the two. We believe, however, that while a retrospective design is not free of bias, it does accurately reflect the patient's current perception of their HRQOL, which, in a clinical setting, is of central importance. Had we not discovered that all of the patients operated on for GD in our study had a $100 \%$ surgical success rate, and had we included patients in the study in whom surgery had failed, the relevance of a "response shift" bias would have been stronger. However, all of the patients in our study had successful surgery, thus guaranteeing a certain homogeneity without having to exclude any patients with unsuccessful surgery. We also believe that our findings are important in light of the fact that surgery is still not often recommended as a first approach in GD patients.

Despite these potential limitations of our study, we observed that surgical therapy resolved the hyperthyroid condition in $100 \%$ of cases, leading to an improvement in the HRQOL of patients in almost all cases examined, especially with regard to the organic/clinical and psychosocial problems, with no deterioration recorded in any patient.

\section{Conclusions}

Surgery should be proposed as an immediate and completely effective solution for GD, especially when compared with prolonged medical therapy, because it can provide a demonstrable improvement in the HRQOL of patients. We think it is important to stress that some preoperative symptoms, such as social isolation, limited working capacity and cardiovascular alterations, seem to resolve better than other symptoms with surgery. Although this was a retrospective study, we think that it may offer important and useful information for the international scientific community, and perhaps contribute to enriching and promoting research in this field, although further studies will have to be carried out to confirm our findings.

Conflict of interest Gregorio Scerrino and co-authors have no conflict of interest; they declare that there is no conflict of interest that could be perceived as prejudicing the impartiality of the research reported and that this research did not receive any specific grant from any funding agency in the public, commercial or not-for-profit sector. They also have full control of all primary data, so they agree to allow the journal to review their data if requested.

\section{References}

1. Heemstra KA, Smit JWA. Advances in the treatment of Graves' disease-a focus on Rituximab. Eur Endocrinol. 2008;4(II).

2. Watt T, Groenvold M, Rasmussen AK, Bonnema SJ, Hegedüs L, Bjorner JB, et al. Quality of life in patients with benign thyroid disorders. A review. Eur J Endocrinol. 2006;154:501-10.

3. Elberling TV, Rasmussen AK, Feldt-Rasmussen U, Hørding M, Perrild H, Waldemar G. Impaired health-related quality of life in Graves' disease. A prospective study. Eur J Endocrinol. 2004; 151:549-55.

4. Ladenson PW. Psychological well-being in patients. Clin Endocrinol. 2002;57:575-6.

5. Chris M, Bruns CM, Chen $\mathrm{H}$. The surgical management of Graves' disease. J Surg Res. 2006;133:207-14.

6. Boostrom S, Richards ML. TT is the preferred treatment for patients with Graves' disease and a thyroid nodule. Otolaryngol Head Neck Surg. 2007;136:278-81.

7. Cooper DS. Antithyroid drugs. N Engl J Med. 2005;352(9): 905-17.

8. Palit TK, Miller CC 3rd, Miltenburg DM. The efficacy of thyroidectomy for Graves' disease. A meta-analysis. J Surg Res. 2000;90(2):161-5.

9. Weber KJ, Solorzano CC, Lee JK, Gaffud MJ, Prinz RA. Thyroidectomy remains an effective treatment option for Graves' disease. Am J Surg. 2006;191(3):400-5.

10. Al-Adhami A, Craig W, Krukowski ZH. Quality of life after surgery for Graves' disease: comparison of those having surgery intended to preserve thyroid function with those having ablative surgery. Thyroid. 2012;22(5):494-500. (Epub 2012 Mar 15).

11. Cawood T, Moriartry P, O'Shea D. Recent developments in thyroid eye disease. BMJ. 2004;329:385-90.

12. Estcourt S, Quinn AG, Vaidya B. Quality of life in thyroid eye disease: impact of quality of care. Eur J Endocrinol. 2011; 164:649-55.

13. Gerding MN, Terwee CB, Dekker FW, Koornneef L, Prummel MF, Wiersinga WM. Quality of life in patients with Graves' ophthalmopathy is markedly decreased: measurement by the medical outcomes study instrument. Thyroid. 1997;7:885-9.

14. Kahaly GJ. Psychological morbidity of Graves' orbitopathy. Clin Endocrinol. 2005;103:368-411.

15. The EuroQol Group. EuroQol-a new facility for the measurement of health-related quality of life. Health Policy. 1990; 199-208. 
16. Bradley EA, Sloan JA, Novotny PJ, Garrity JA, Woog JJ, West SK. Evaluation of the national Eye Institute Visual Function Questionnaire in Graves' ophthalmopathy. Ophthalmology. 2006;113:1450-4.

17. Farid M, Roch-Levecq AC, Levi L, Brody BL, Granet DB, Kikkawa DO. Psychological disturbance in Graves' ophthalmopathy. Arch Ophthalmol. 2005;123:491-6.

18. Terwee CB. Long-term effects of Graves' ophthalmopathy on health-related quality of life. Eur J Endocrinol. 2002;146:751-7.

19. R Development Core Team. R statistical software package, version 2.2.0, 2005. Available at: http://www.r-project.org.

20. Takamura Y, Nakano K, Uruno T, Ito Y, Miya A, Kobayashi K, et al. Changes in serum TSH receptor antibody (TRAb) values in patients with Graves' disease after total or subtotal thyroidectomy. Endocr J. 2003;50(5):595-601.

21. Laurberg P, Wallin G, Tallstedt L, Abraham-Nordling M, Lundell G, Tørring O. TSH-receptor autoimmunity in Graves' disease after therapy with anti-thyroid drugs, surgery, or radioiodine: a 5-year prospective randomized study. Eur $\mathrm{J}$ Endocrinol. 2008;158(1):69-75.

22. Schüssler-Fiorenza CM, Bruns CM, Chen H. The surgical management of GD. J Surg Res. 2006;133(2):207-14. (Epub 2006 Feb 3).
23. Kashiwai T, Hidaka Y, Takano T, Tatsumi KI, Izumi Y, Shimaoka Y, Tada H, Takeoka K, Amino N. Practical treatment with minimum maintenance dose of anti-thyroid drugs for prediction of remission in Graves' disease. Endocr J. 2003;50(1):45-9.

24. Weetman AP. Controversy in thyroid disease [comment] [Review; 44 refs]. J R Coll Physicians Lond. 2000;34:374.

25. Wiersinga WM, Bartalena L. Epidemiology and prevention of Graves' ophthalmopathy. Thyroid. 2002;12:855-60.

26. Whybrow PC, Bauer M. Behavioral and psychiatric aspects of thyrotoxicosis. In: Braverman LE, Utiger RD, editors. Werner \& Ingbar's the thyroid: a fundamental and clinical text. 8th ed. Philadelphia: Lippincott Williams \& Wilkins; 2000. p. 673-8.

27. Benevicius R, Velickiene D, Prange AJ Jr. Mood and anxiety disorders in women with treated hyperthyroidism and ophthalmopathy caused by Graves' disease. Gen Hosp Psychiatry. 2005;27:133-9.

28. Coulter I, Frewin S, Krassas GE, Perros P. Psychological implication of Graves' orbitopathy. Eur J Endocrinol. 2007;157: $127-31$.

29. Nieuwkerk PT, Tollenaar MS, Oort FJ, Sprangers MA. Are retrospective measures of change in quality of life more valid than prospective measures? Med Care. 2007;45(3):199-205. 